\title{
Beneficios psicosociales de los grupos de apoyo: su influencia en los estilos de socialización familiar
}

\section{Psychosocial benefits of the support: their influence in the family socialization styles}

\section{M. - Teresa VEGA RODRÍGUEZ* M. - Yolanda DE DIOS DE DIOS*}

\section{RESUMEN}

En los últimos años los programas de Escuela de Padres se han convertido en una estrategia de promoción de salud en los hijos. El grupo de padres se convierte en un medio eficaz de cambio social al ser fuente de comprensión, expresión de sentimientos y apoyo. El objetivo de este estudio es analizar la influencia que la integración grupal tiene en el autoconcepto familiar y cómo incide en las pautas de socialización. La muestra está compuesta por 190 progenitores a los que se aplicaron medidas de autoinforme para evaluar la integración grupal (sentido de grupo), el autoconcepto familiar (autoeficacia como agente preventivo, autoestima familiar, satisfacción con el rol parental desempeñado) y los estilos educativos (comunicativo, de apoyo). En los resultados se constata que el sentido de grupo optimiza los recursos psicosociales. Los progenitores se sienten capacitados para crear un clima familiar que les ayude a poner en práctica las pautas de socialización más apropiadas y convertirse asi en agentes de salud efectivos.

\section{PALABRAS CLAVE} ción.

Socialización familiar, Influencia grupal, Autoeficacia, Autoestima, Apoyo y comunica-

\footnotetext{
* Departamento de Psicologia Social y Antropologia. Facultad de Psicología. Universidad de Salamanca.
} 
Beneficios psicosociales de los grupos de apoyo: su influencia en los estilos de socialización familiar

\begin{abstract}
In recent years programmes for parents have become a strategy for the promotion of health in children. The group becomes an effective medium of social influence by being a source of understanding, expression of feelings and support. The objective of the study is to test the effect that group integration has on the family self-concept and to find out the impact it has on socialization patterns. The sample comprises 190 parents who were given a self-report that measured the sense of group, self-concept (self-efficacy as a preventive agent, family self-esteem, satisfaction with the role played) and educational styles (communicative, supporting). The results show that the sense of group optimises the psychosocial resources that advocate parents becoming effective agents for change, since they feel able to create the family conditions that help parents to put into practice the most suitable socialization patterns.
\end{abstract}

\title{
KEY WORDS
} cation.

Family socialization, Group influence, Self-efficacy, Self-esteem, Support and communi- 


\section{BENEFICIOS FAMILIARES DE LOS GRUPOS DE APOYO}

La familia representa el escenario natural y cotidiano de promoción de la salud. Son numerosos los estudios que demuestran la relación significativa entre factores familiares de riesgo como el aislamiento social y las normas parentales inconsistentes, y consumos abusivos de drogas, prácticas sexuales de riesgo de los hijos, etc. (De Dios, Vega y Ramírez, 2001; Gracia, Musitu y García, 1990; Musitu y Lila, 1993; Pons y Berjano, 1997; Vega y Garrido, 2000). Este aspecto justifica el uso de estrategias de intervención orientadas a trabajar con los padres en grupos de apoyo.

El grupo de progenitores se convierte en un contexto significativo de influencia socio-familiar. La experiencia grupal que promueve el sentido de grupo (cfr. Sarason, 1974) contribuye al bienestar de los progenitores al favorecer percepciones más positivas de sí mismos y mejorar las interacciones padres-hijos que, en muchas ocasiones, están en la base de los conflictos familiares.

Estos beneficios grupales responden a dos claves de éxito. La primera, basada en la Teoría de la Identidad Social (Tajfel, 1984), se refiere a las necesidades compartidas por los miembros del grupo que facilitan la identificación social: buscar estrategias para resolver conflictos, mejorar el control y dominio de la realidad, obtener consejo (Ayestarán, MartínezTaboada y Arróspide, 1996; Díaz y Ferri, 2002; Martínez-Taboada, 1996).

La segunda clave de éxito radica en que las relaciones de intercambio de información sociocognitiva (por ejemplo, cuando en sus conversaciones transmiten conocimientos personales al grupo) y de aceptación y comprensión mutua (Solomon, Pistrang y Barker, 2001) que se establecen entre los progenitores, les capacitan para definir más claramente su situación y las vías de solución (Schubert y Borkman, 1994).

\section{AUTOCONCEPTO FAMILIAR Y ESTILOS DE SOCIALIZACIÓN}

La integración en el grupo de iguales, siguiendo a Cohen (1988), facilita modelos de conducta a partir de los cuales los progenitores infieren sentimientos de autoconfianza y competencia (Bandura, 1987, 1999) y desarrollan la percepción de control de la situación familiar, asumiendo su responsabilidad en la solución de los problemas. Esto es importante puesto que ser padre 0 madre requiere tener seguridad en las propias capacidades para abordar con confianza los desafíos educativos, es decir, tener la creencia de eficacia personal (Bandura, 1999).

La autoeficacia personal representa, como afirma Maddux (1991), la actitud hacia uno mismo y la valoración de las propias capacidades para manejar con éxito las situaciones familiares, laborales, etc. a las que se enfrenta el sujeto. $\mathrm{Su}$ función de mediador sociocognitivo ha sido probada en diversas investigaciones (véase Bandura, 1999). En la evaluación de resultados del programa de Escuela de Familias (EFF) planteada por Vega (1998), se pone de relieve que los padres con mayor grado de eficacia parental, en comparación con los de menor grado de eficacia, responden adecuadamente a la conducta comunicativa de los hijos, les proporcionan apoyo afectivo y material y logran crear las condiciones familiares necesarias para transmitir expectativas de capacidad que favorezcan el desarrollo integral de los menores. Consiguientemente, los progenitores que piensan que pueden intervenir en la conducta de los hijos son los que más se 
esfuerzan por lograr sus propósitos como educadores.

Al igual que ocurre con la autoeficacia, la autoestima en el dominio familiar es un recurso psicosocial deseable. Para Bandura (1987) y Maddux (1991), el primero conlleva la estimación de la capacidad personal (cognición) y, el segundo, el juicio de la valía personal (afecto). Representa para Lila, Musitu y Molpereces (1994) la actitud evaluativa de aprobación personal y la satisfacción del individuo consigo mismo. Los progenitores con alta autoestima se perciben valorados por los hijos, la pareja, los amigos y otras personas significativas. Baumeister (1991) explica que las personas con mayor autoestima, en comparación con las de menor valia, tienden a persistir en sus esfuerzos ante situaciones de fracaso $\mathrm{y}$ a interpretar sus intentos frustrados como motivos de lucha. Además, la autoestima familiar predispone la actitud de ayudar a los hijos (Herrero, 1996) y facilita la expresión de sentimientos positivos hacia los mismos.

Las creencias de eficacia y autoestima de los padres desempeñan un papel determinante de los estilos educativos familiares, definidos como estrategias utilizadas para regular la conducta y transmitir valores y normas (Lila y Marchetti, 1995), al fomentar conductas de atención y cuidado apropiadas. Los padres que se perciben eficaces y valorados se caracterizan por practicar un estilo autorizativo (Baumrind, 1983), por orientar y guiar las actividades de los hijos de forma racional y centrar sus actuaciones en la resolución del problema. Los estudios apoyan esta linea argumental al constatar que las prácticas basadas en estilos comunicativos y de apoyo aumentan la capacidad de influencia y control sobre los hijos y la posibilidad de que adquieran y mantengan estilos de vida saludables.
Comunicar debidamente las reglas familiares es un ejercicio trascendental, puesto que informan al joven de cómo actúa la familia y de cómo debe actuar él o ella dentro y fuera del entorno familiar. Sin embargo, la comunicación padres-hijos suele ser pobre, predominando un estilo educativo autoritario basado en una comunicación deficitaria y unilateral (Méndez, 1998). El estudio de Garrido y Vega (1996) constata la falta de comunicación paterno-filial al comprobar que los padres no tienen expectativas ajustadas a la realidad del consumo de tabaco y alcohol de los hijos. Al igual, Amato y Ochiltree (1986) descubren que las relaciones familiares en las que predomina una escasa comunicación entre los miembros, se asocian con un autoconcepto negativo y con problemas de competencia social en los hijos.

Practicar un estilo de apoyo socioemocional ajustado a las circunstancias y demandas evolutivas del neófito y caracterizado por compartir sentimientos y pensamientos con los menores y por hacerles ver que entienden y comparten sus sentimientos, es una conducta parental que refuerza actitudes y conductas preventivas deseables en los hijos. Representa un estilo educativo democrático caracterizado por afecto, comprensión y apoyo (Méndez, 1998). Este planteamiento es confirmado por Amato y Ochiltree (1986) al comprobar que las interacciones familiares que ofrecen apoyo emocional a sus componentes, se asocian con un alto nivel de identidad y con un autoconcepto positivo en adolescentes y adultos.

\section{OBJETIVOS}

El objetivo principal es analizar si los padres que se sienten más integrados socialmente y desarrollan un sentido de 
grupo (como proceso de influencia social), incrementan sus expectativas de eficacia y sentimientos de autovalía parentales y experimentan cambios en la conducta de interacción padres- hijos, en comparación con los que se sienten menos integrados (véase figura 1).

\section{DESCRIPCIÓN DEL PROGRAMA EDUCATIVO PARA PADRES}

El objetivo general es realizar una intervención que incremente las estrategias formativas de los padres de forma que se conviertan en agentes educativos eficaces.

Figura 1. Efectos del sentido de grupo sobre los estilos de socialización

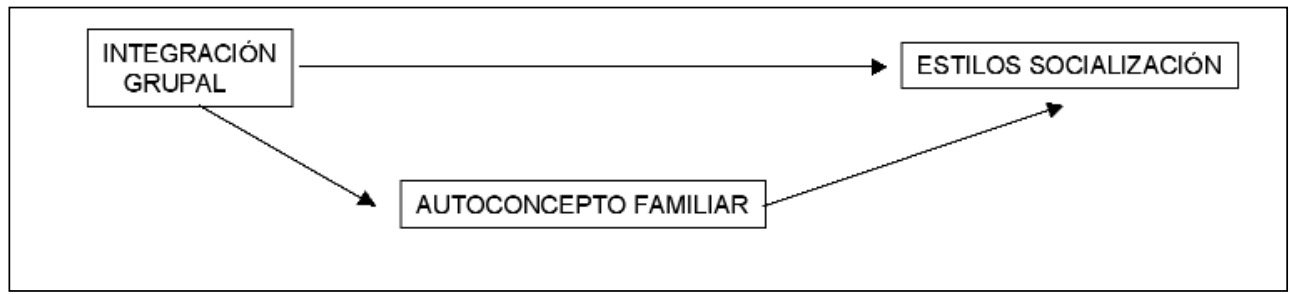

Como objetivo específico se analiza la influencia mediadora del autoconcepto familiar entre el sentido de grupo y los estilos parentales.

\section{MÉTODO}

\section{Muestra}

Participaron 190 progenitores cuyos hijos asistían a clase en 23 centros educativos de enseñanza obligatoria de Salamanca capital. El 61\% eran madres y el porcentaje restante padres. El intervalo de edad oscila entre 22 y 65 años, siendo la edad media de 38 años. La mayoría de los progenitores (53\%) han cursado estudios primarios y el 1,2\% carecen de estudios. El 69,8\% de los participantes no tiene trabajo remunerado (trabajan en las tareas del hogar o están en paro).

Respecto al tipo de familia, el 92,7\% de los participantes se constituyen en familias con dos progenitores sea en situación de matrimonio, familia reconstituida o convivencia. Las familias tienen una media de 1,90 hijos, oscilando el número de hijos de 1 a 7 .
Los objetivos específicos pretenden que los padres:

- Incrementen su sentido de competencia y su capacidad para la resolución de problemas.

- Reflexionen acerca de su tarea educativa y de los factores de riesgo familiares y sociales.

- Fomenten su autoestima y la de sus hijos.

- Faciliten un buen cauce de comunicación familiar.

Los temas abordados se centran en los ciclos evolutivos de la familia, psicología evolutiva, autoestima, comunicación, habilidades parentales, educación sexual, prevención de drogodependencias y cualquier otro que los padres sugieran.

Se desarrollan 10 sesiones quincenales de hora y media de duración, distribuidas entre los meses de noviembre y marzo. Las sesiones constan de una introducción teórica, seguida de dinámicas de grupo y finalizan con una evalua- 
ción de la sesión por parte de los asistentes y de los educadores.

\section{PROCEDIMIENTO}

Se contactó con los padres y madres a través de la oferta del programa realizada por el Plan Municipal sobre Drogodependencias del Ayuntamiento de Salamanca a los centros educativos. Al comenzar el curso académico se convocó una reunión con el equipo directivo y con el AMPA de cada centro en la que se explicaron las características del programa y se solicitó su participación. Se aprovechó esta reunión para pedir su colaboración voluntaria en esta investigación mediante la respuesta a un cuestionario.

La administración colectiva del cuestionario se realizó en la última sesión del programa respetando la distribución en grupos de 8 a 10 personas, uno por cada centro escolar. Los cuestionarios eran aplicados por los educadores quienes explicaban las caracteristicas del mismo, forma de respuesta y aseguraban el anonimato y confidencialidad. Los educadores fueron previamente instruidos por los investigadores sobre el contenido y la forma de aplicación.

\section{INSTRUMENTOS DE MEDIDA}

El cuestionario constaba de diferentes escalas referidas a las siguientes variables:

- Sentido de grupo. Valoración que los asistentes hacen de los otros padres como fuente de comprensión, seguridad, expresión de sentimientos y actitudes positivas. Se utilizaron cuatro items basados en la definición de sentido de comunidad de Sarason (1974) pero aplicado al grupo. El patrón de respuesta iba de muy en desacuerdo a muy de acuerdo, una mayor puntuación indicaba un mayor grado de sentido de grupo. Los cuatro items constituian un factor con una consistencia interna de 0,78.

- Autoconcepto familiar. Para medir este constructo se utilizaron tres tipos de indicadores: uno de carácter sociocognitivo, la autoeficacia de desempeño del rol de madre/padre, otro de tipo afectivo, la autoestima familiar y un último de tipo cognitivo-evaluativo, la satisfacción como padres.

1) Autoeficacia parental. Grado de seguridad con que los padres y madres se creen capaces de poder superar las dificultades que surgen con sus hijos y de transmitirles autoeficacia. Se utilizaron cuatro items especificamente diseñados para esta investigación. Los participantes respondian en una escala de confianza que iba de 0 (no estoy nada seguro/a) a 4 (estoy muy seguro/a). El alpha de Cronbach fue de 0,74.

2) Autoestima familiar. Juicio y valoración personal de los progenitores acerca de cómo perciben su relación con la familia y de cómo le valora. Los participantes expresaban su grado de acuerdo utilizando una escala tipo Likert que iba de muy en desacuerdo (1) a muy de acuerdo (5). Cuatro de los seis items de los que consta la escala fueron adaptados de la medida de autoestima familiar de Herrero, Gracia y Musitu (1996). La consistencia de la escala fue de 0,84.

3) Satisfacción parental. Grado en el que los padres se sienten satisfechos con cómo desempeñan su rol de educadores. El patrón de respuesta iba de no estoy nada satisfecho/a (0) a estoy muy satisfecho/a (4). La fiabilidad de los tres items diseñados por Vega (1998) fue de 0,73.

- Estilo conductual. Se utilizaron 
dos estilos de socialización para medir esta variable:

1) Comunicativo. Proceso de comunicación que se desarrolla entre padres e hijos: si hablan abiertamente, si tratan de comprenderlos o intentan razonar con ellos. El patrón de respuesta iba de muy en desacuerdo (1) a muy de acuerdo (5). Se utilizó la escala de estilo comunicativo diseñada por Vega (1998). La consistencia interna fue de 0,79 .

2) De apoyo. Las dos dimensiones medidas representan las principales funciones que cumple el apoyo. Algunos items pertenecen a la escala de apoyo elaborada por García (1994). Los padres señalaban el grado de acuerdo de 1 a 5 .

a) Emocional: Actitudes comprensivas y muestras de cariño, amor, agrado, empatía que los progenitores proporcionan a los hijos. Los 7 items forman una escala con un alpha de Cronbach de 0,83 .

b) Instrumental e informacional. Ayuda material que los progenitores ofrecen a sus hijos, así como información y consejo. La escala tiene una consistencia interna de 0,84

\section{RESULTADOS}

Para validar el path análisis de la figura 1 y verificar las condiciones de mediación se siguió el procedimiento de Baron y Kenny (1986) según el cual, para valorar el efecto mediador de una variable, se tiene que demostrar que el sentido de grupo: 1) está asociado al autoconcepto. 2) se relaciona con los estilos de socialización. 3) no se relaciona con los estilos de socialización cuando está presente el autoconcepto. Por ello se realizaron seis ecuaciones de regresión múltiple jerárquica. La ecuación estructural que representa cada predictor (autoconcepto y estilos de socialización) tenía como antecedentes la variable explicativa exógena (sentido de grupo) y las endógenas (autoestima y autoeficacia) que teóricamente le influian. $\mathrm{El}$ género no fue tenido en cuenta como covariable dada la escasa asociación que mantenía con las otras variables.

En la figura 2 se exponen los coeficientes de regresión estandarizados significativos. Los resultados ponen de manifiesto que el sentido de grupo influye significativamente en la autoeficacia $\left(\mathrm{R}^{2}{ }_{(1,175)}=0,04, p \leq 0,01\right)$ y autoestima $\left(\mathrm{R}^{2}{ }_{(2,169)}=0,23, p \leq 0,001\right)$ pero no en la satisfacción parental. También se halla que el sentido de grupo afecta de forma relevante al estilo comunicativo $\left(\mathrm{R}_{(4,158)}^{2}=0,30, p \leq 0,001\right)$.

Se constata que la autoeficacia y la autoestima familiares median el impacto del sentido de grupo sobre el apoyo social y promueven estilos de apoyo social emocional $\left(\mathrm{R}^{2}{ }_{4,153)}=0,44, p \leq 0,001\right)$ e instrumental $\left(\mathrm{R}^{2}(4,152)=0,35, p \leq 0,001\right)$. El valor de los coeficientes pone de relieve que la autoestima ejerce mayor efecto mediador y tiene mayor poder explicativo del apoyo social que la percepción de la eficacia. Se constata que la satisfacción familiar no explica los patrones de conducta parental.

En el path análisis se muestra que la autoeficacia familiar aumenta la percepción de autovalía de los padres, haciendo que se sientan orgullosos, útiles y valorados por la familia. Se constata que la percepción de capacidad no sólo funciona como un determinante de los estilos de socialización sino que, además, influye en ellos a través de la autoestima familiar. A su vez se observa que, a mayor nivel de confianza personal y de autovaloración positiva, aumenta el grado de satisfacción que presentan con la forma 
que tienen de educar a los hijos y con cómo combinan sus tareas extra-familiares con las familiares. como variables intervinientes que condicionan las acciones de los progenitores.

\section{Figura 2. Path análisis de la influencia que ejerce en el sentido de grupo sobre} los estilos parentales

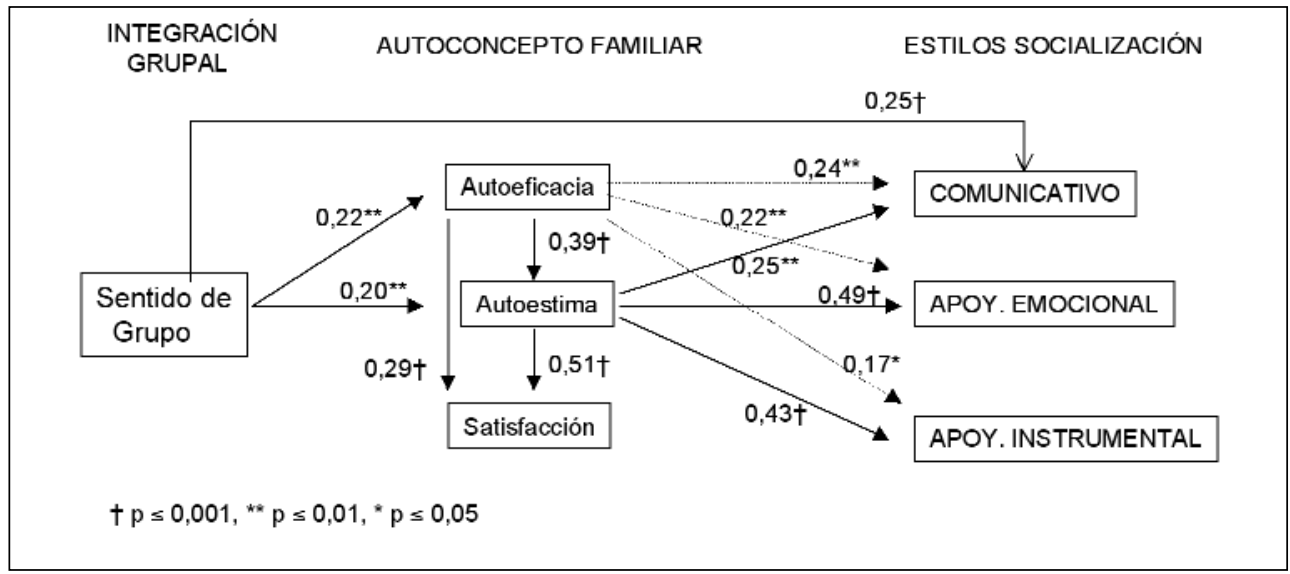

En el caso del estilo comunicativo no se apoya el efecto mediador del autoconcepto, al ejercer la integración grupal una influencia directa y significativa sobre este estilo $(=0,25, p<0,001)$. Se demuestra que estar integrado en el grupo de EFF mejora directamente el estilo de comunicación padres-hijos percibido por los progenitores, de forma que aquellos que se sienten más parte del grupo reconocen que les prestan más atención a sus hijos y tratan más de razonar con ellos.

\section{DISCUSIÓN Y CONCLUSIONES}

Los análisis presentados aportan pruebas empíricas de que el sentido de grupo repercute en el estilo de interacción paterno-filial por medio de procesos autoevaluativos cognitivo-afectivos. Este resultado apoya los principios de la Teoría Social Cognitiva formulada por Bandura (1987) al confirmar que la autoeficacia y la autoestima funcionan
Los grupos, al actuar como marco de referencia psicológico, capacitan a los padres para definirse a sí mismos más positivamente y atribuir significados más positivos a sus interacciones con los hijos. Levy (1979) considera que los grupos de apoyo hacen emerger una cultura alternativa al hacer que los miembros desarrollen nuevas definiciones de sus identidades personales y normas en las que basar su autoestima.

La práctica de compartir experiencias con otros progenitores genera conocimiento experiencial válido sobre la naturaleza del problema, que les capacita para identificar cuáles pueden ser las estrategias más efectivas para abordar las demandas y exigencias de sus hijos y para anticipar las consecuencias que una $\mathrm{u}$ otra actuación podrían llegar a producir. Aquellos padres que se perciben más autoeficaces para afrontar efectivamente los retos educativos son los más capacitados para anticiparlos al intentar modificar la conducta de los hijos. Se constata 
pues, que el grupo social constituye una fuente de autoeficacia, puesto que en la medida en que se produce la identificación social el grupo adquiere credibilidad y poder de persuasión social.

La identificación social ha favorecido la autoestima familiar al hacer comprender a los padres que las dificultades diarias con los hijos son problemas comunes y resolubles. Al escuchar de otros preocupaciones similares a las suyas, al sentirse comprendidos y al adquirir una idea más clara de lo que representa su rol, toman conciencia de que sus conflictos no son singulares, sino que forman parte de la dinámica familiar. Ello hace que los padres aumenten su valoración positiva como educadores y piensen que sus hijos, a pesar de su desobediencia, les respetan $\mathrm{y}$ valoran los esfuerzos que hacen por ellos. Tal razonamiento está en consonancia con la Teoria de la Atribución Social, ya que conocer que las preocupaciones propias son compartidas, disminuye la gravedad percibida, el carácter amenazante de la situación (cfr. Wills, 1985) y da lugar a una autoimagen más positiva de sî mismos como padres (Gil y García, 1996).

En suma, la aprobación de los demás tiene poder recompensante y motiva los esfuerzos necesarios para alcanzar los objetivos familiares propuestos. Este aspecto evidencia la influencia normativa que ejerce el grupo social (Deutsch y Gerard, 1955). La incertidumbre que los padres tienen sobre sus capacidades y rendimientos la solventan comparándose con los demás. En la comparación utilizan estándares externos basados en las creencias y opiniones consensuadas que se expresan en las sesiones grupales, las cuales confirman la deseabilidad o indeseabilidad de conductas paterno-filiares concretas.

La integración en el grupo no ejerció un efecto determinante sobre la satisfac- ción familiar y sí lo hicieron la autoestima y la autoeficacia. Esto evidencia que la satisfacción depende más de qué piensan y sienten los progenitores que de lo que realmente obtienen con sus esfuerzos. Es como si sus experiencias en el grupo les hubieran demostrado que el ser persistente e intentar distintas soluciones es tan importante o más que el resultado final, puesto que éste no sólo depende de la actuación de los padres sino también de las influencias de otros contextos (escuela, medios de comunicación, ...). Por tal motivo, intentar hacer las cosas bien, aunque no salgan como uno desee, es fuente de orgullo y satisfacción en sí misma. Este resultado es confirmado por Bandura (1987) al demostrar que la implicación en el logro de metas también determina la satisfacción personal. El razonamiento expuesto es aún más comprensible si tenemos en cuenta que no existen criterios claros y objetivos para enjuiciar qué es ser un buen padre 0 madre, los cuales sí existen para definir a un mal padre o madre.

Este argumento explicaría también el efecto positivo y directo de la autoeficacia parental sobre la autoestima y la satisfacción. La recompensa de los padres autoeficaces está, sobre todo, en el hecho de haberse esforzado en intentar mejorar como padres.

El que la autoestima ejerza mayor influencia positiva sobre el apoyo social que sobre el estilo comunicativo podria explicarse atendiendo al carácter emocional y afectivo de la autoestima y del apoyo. Los padres con mayor autoestima familiar transmiten más fácilmente sus sentimientos y emociones y son más sensibles a este tipo de necesidades en los hijos. Son también los que desarrollan más comportamientos de ayuda, confian más en ellos y tratan de que sean más independientes y autónomos. 
Los resultados expuestos indican que para potenciar estilos comunicativos y de apoyo social, los padres necesitan tener una alta autovalía y confianza en sus propias capacidades. Ambos aspectos son necesarios para crear situaciones que favorezcan el diálogo y el apoyo a los hijos y para interpretar más positivamente sus reacciones a estos intentos.

En el análisis de vías se pone de manifiesto que la satisfacción de los progenitores respecto a cómo están desarrollando su labor no afecta a los estilos de socialización. Esto se explica si apelamos a un argumento ya razonado: los estilos de socialización familiar, al no producir cambios inmediatos en la conducta de los hijos, hacen que la conducta de interacción paterno-filiar esté más determinada por los resultados anticipados que por los inmediatos. Motivo por el cual la percepción de capacidad y la percepción de la valoración familiar, y no la satisfacción derivada de la obtención de resultados, determinen los estilos disciplinares. Otra explicación es que quizás la satisfacción familiar puede estar actuando como consecuencia de los estilos educativos y no como promotor de los mismos, ya que la realización de la conducta es fuente de satisfacción personal.

La influencia directa del grupo en la conducta comunicativa confirma el modelo de Influencia Informativa de Deutsch y Gerard (1955). Ante la ausencia de criterios objetivos y contrastables, los padres, para evaluar la validez de sus opiniones y habilidades de comunicación, aceptan la visión que los otros miembros puedan aportar acerca de la realidad y, a raíz de ello, reorientan su conducta de interacción (Campbell, Tesser y Fairey, 1986).

El que el sentido de grupo y los recursos psicológicos potencien disciplinas basadas en el diálogo, la ayuda y la transmisión de sentimientos, muestra que los padres han sido capaces de adoptar estilos de socialización activos tales como el estilo autorizativo planteado por Baumrind (1983) o la disciplina inductiva o de apoyo planteada por Musitu y Gutiérrez (1984).

Una última cuestión a plantear es porqué el sentido de grupo produce un efecto directo sobre el estilo comunicativo y no sobre los estilos de apoyo social. Una explicación sería que para los padres y madres, la conducta comunicativa es más dificil de evaluar que las conductas de apoyo y, por ello, el efecto persuasivo del grupo es más potente.

En líneas generales, los resultados discutidos ponen de relieve que el grupo de EFF es un recurso social significativo que favorece el funcionamiento familiar. Asimismo, demuestran que la participación en el grupo potencia los recursos psicosociales básicos que los padres necesitan para actuar como agentes activos de cambio de su conducta de interacción padres-hijos. En suma, las prácticas parentales se pueden modificar siempre que se logren alterar las percepciones y autovaloraciones de los padres que potencian mayor control personal de las condiciones familiares. 


\section{BIBLIOGRAFÍA}

Amato, P.R. y Ochiltree, G. (1986). Family resources and the development of child competence. Journal of Marriage and the Family, 48, 4756.

Ayestarán, S., Martínez-Taboada, C. y Arróspide, J.J. (1996). La teoría psicosocial del grupo. En S. Ayestarán (ed.), El grupo como construcción social (189-214). Barcelona: Plural.

Bandura, A. (1987). Pensamiento y acción. Fundamentos Sociales. Barcelona: Martínez Roca.

Bandura, A. (ed.) (1999). Autoeficacia: Cómo afrontamos los cambios de la sociedad actual. Bilbao: Desclée de Brouwer.

Baron, R.M. y Kenny, D.A. (1986). The moderator-mediator variable distinction in social psychological research: conceptual, strategic, and statistical considerations. Journal of Personality and Social Psychology, 51 (6), 1173-1182.

Baumeister, R.F. (1991). Self-concept and identity. En V.J. Derlega, B.A. Winstead, y W.H Jones (eds.), Personality Contemporary Theory and Research (349-380). Chicago: Nelson-Hall publishers.

Baumrind, B.D. (1983). Familial antecedents of adolescent drug use: a developmental perspective. En C. La Rue-Jones y R. Battjes (eds.), Etiolo gy of drug abuse: implications for prevention (1340). National Institute of Drug Abuse Research Monograph, 56.

Campbell, J.D., Tesser, A. y Fairey, P.J. (1986). Conformity and attention to the stimulus: some temporal and contextual dynamics. Journal of Personality and Social Psychology, 51, 315-324.

Cohen, S. (1988). Psychosocial models of the role of social support in the etiology of physical disease. Health Psychology, 7, 269-297.

De Dios, Y., Vega, M.T. y Ramírez, R. (2001). Aplicación de un programa de Escuela de Familias y su evaluación: Manual de trabajo. Salamanca: Plan Nacional Sobre Drogas y Ayuntamiento de Salamanca.

Deutsch, M. y Gerard, H.B. (1955). A study of normative and informational social influence upon individual judgment. Journal of Abnormal and Social Psychology, 51, 629-636.
Díaz, R. y Ferri, M.J. (2002). Intervención en poblaciones de riesgo (1): los hijos de alcohólicos. Adicciones. Revista de Socidrogalcohol, 14 (1 sup.), 353-374.

García, F. (1994). Evaluación de la socialización familiar. En G. Musitu y P. Allat, Psicosociología de la familia (295-320). Valencia: Albatros.

Garrido, E. y Vega, M.T. (1996). Conducta de fumar $y$ beber en los estudiantes de $7^{\circ}$ y $8^{\circ}$ de E.G.B. Informe no publicado. Ayuntamiento de Salamanca.

Gil, F. y García, M. (1996). Los procesos de influencia social en el grupo. En S. Ayestarán (ed.), El grupo como construcción social (103-132). Barcelona: Plural.

Gracia, E., Musitu, G. y García, F. (1990). El apoyo social: una variable moduladora en las relaciones padres-hijos en situaciones de maltrato. III Congreso Nacional de Psicología Social (Vol. 1, 225-237). Santiago de Compostela.

Herrero, J. (1996). Autoestima y sistemas informales de apoyo: su efecto en el bienestar. Informació Psicològica, 61, 21-27.

Herrero, J., Gracia, E. y Musitu, G. (1996). Salud y comunidad. Evaluación de recursos y estresores. Valencia: Conselleria de Cultura, Educació y Ciencia de la Generalitat Valenciana.

Levy, L. (1979). Processes and activities in groups. En M. Lieberman, y L. Borman (Eds.), Selfhelp groups for coping with crisis: origins, members, processes and impact (234-271). San Francisco: Jossey-Bass.

Lila, M.S y Marchetti, B. (1995). Socialización familiar: valores y autoconcepto. Informació Psicológica, 59, 11- 17.

Lila, M.S., Musitu, G. y Molpereces, M.A. (1994). Familia y autoconcepto. En G. Musitu y P. Allat, Psicosociología de la familia (83-103). Valencia: Albatros.

Maddux, J.E. (1991). Personal efficacy, En J. Derlega, B.A. Winstead y W.H. Jones, Personality. Contemporary theory and research (231-261). Chicago: Nelson-Hall publishers.

Martínez-Taboada, C. (1996). Estudio de los 
grupos en el área de la salud. En S. Ayestarán (ed.), El grupo como construcción social (215-232). Barcelona: Plural.

Méndez, F. X. (1998). El niño que no sonríe: estrategias para superar la tristeza y la depresión infantil. Madrid: Pirámide.

Musitu, G. y Gutiérrez, M. (1984). Disciplina familiar, rendimiento y autoestima. Actas Jornadas Nacionales de Orientación Profesional.

Musitu, G. y Lila, M. (1993). Estilos de socialización e intensidad de la comunicación en las relaciones padres-hijos. Revista de Psicología, 15 (2), $15-29$.

Pons, J. y Berjano, E. (1997). Análisis de los estilos parentales de socialización asociados al abuso de alcohol en adolescentes. Psicothema, 9 (3), 609-617.

Sarason, S. (1974). The psychological sense of community: prospects for a community psychology. San Francisco: Jossey-Bass.

Solomon, M., Pistrang, N. Y Barker, C. (2001) The benefits of mutual support groups for parents of children with disabilities. American Journal of Community Psychology, 29 (1), 113- 132.

Schubert, M.A. y Borkman, T. (1994). Identifying the experiential knowledge developed within a self- help group. En T. J. Powell, Understanding the self- help organization: frameworks and findings (227- 246). California: Sage.

Tajfel, H. (1984). Categorización social, identidad social y comparación social. En H. Tajfel, Grupos humanos y categorías sociales (291-304). Herder: Barcelona.

Vega, M.T. (1998). Evaluación de un programa municipal de Escuela de Familias. Ayuntamiento de Salamanca. Informe técnico no publicado.

Vega, M.T. y Garrido, E. (2000). Valoración de una intervención preventiva del consumo adolescente de tabaco y alcohol: incidencia de factores personales y situacionales. Revista de Psicología Social Aplicada, 10 (1), 5-29.

Wills, T.A. (1985). Supportive functions of interpersonal relationships. En S. Cohen y L.C. Syme (eds.), Social support and health. Nueva York: Academic Press. 